\title{
An Overview of Systematic Reviews Related to Aspects of the Shortened Dental Arch and Its Variants in Adults
}

\author{
Saadika B. Khan, BChD, PDD, MSc (Dent)'/Usuf M.E. Chikte, BChD, DHSM, Mdent, MSc, $\mathrm{PhD}^{2}$ / \\ Ridwaan Omar, BSc, BDS, $\mathrm{MSc}^{3}$
}

\begin{abstract}
Purpose: The aim of this study was to conduct an overview of systematic reviews (SRs) related to aspects of the shortened dental arch (SDA) and its variants and critically appraise the methodologic quality of included SRs using the AMSTAR checklist. Materials and Methods: A comprehensive computerized search and a hand search of reference lists were conducted for SRs related to SDAs to identify publications from 2000 to 2016. All the present authors and a research assistant independently screened the results of the electronic searches using an eligibility form and extracted information using a specially designed prepiloted data extraction form. An 11-question AMSTAR checklist was completed for each included SR. Disputes were resolved by discussion between all researchers, and results were collated and interpreted.

Results: For the period of 2007 to 2016, the search yielded nine SRs incorporating 228 related articles. The research questions for each SR differed but were related to SDAs, thus, the included articles were similar across SRs. Characteristics such as aims/objectives, study outcomes, and conclusions of the nine included SRs were compared. The AMSTAR evaluation indicated that five out of nine studies were of a high quality (used a rigorous methodology) and the remaining four were of medium quality. All nine SRs provided designs and characteristics of included studies. None of the SRs assessed publication bias. Conc/usion: Of the nine SRs, seven drew positive conclusions regarding the SDA concept, finding it functionally sound, although some suggested that more high-quality primary studies are still needed. The AMSTAR calculation indicated that most included SRs had an acceptable methodologic quality, emphasizing the reliability of their results. Int J Prosthodont 2017 (10 Pages). doi: 10.11607/ijp.5287
\end{abstract}

$\mathrm{T}$ ranslation and clinical implementation of even the most compelling research evidence takes a long time. For example, it took more than 20 years before the documented evidence for using intravenous streptokinase for the management of acute myocardial infection became the norm. ${ }^{1}$ Similarly, although ample evidence is available for the benefits of the shortened dental arch (SDA) approach as a viable treatment option for a number of population groups, translation into clinical practice is noticeably lacking in these settings. ${ }^{2,3}$ The reasons for this are not fully understood, although undergraduate curricula and syllabi, educational backgrounds and beliefs of clinical teachers, and societal factors play roles. ${ }^{3}$ Implementation

\footnotetext{
'Doctor, Department of Restorative Dentistry, Faculty of Dentistry, University of the Western Cape, South Africa.

2 Professor, Department of Community Health, Faculty of Health Sciences, University of Stellenbosch, South Africa.

${ }^{3}$ Professor, Department of Prosthodontics, Faculty of Dentistry, Kuwait University, Kuwait.
}

Correspondence to: Dr S.B. Khan, Department of Restorative Dentistry, University of the Western Cape, Private Bag X01, Tygerberg 7505, South Africa. Fax : +2721 9382281. Email:skhan@uwc.ac.za

C2017 by Quintessence Publishing Co Inc of the SDA concept may be further compromised by the fact that it can be a financial disincentive. ${ }^{2,3}$ What cannot be contested regarding the SDA is that much of the primary research on it has documented favorable functional efficacy and patient satisfaction.

At about the same time as Käyser's ${ }^{4}$ (1981) formulation of the SDA concept, a strategy of "the retention of 20 functional natural teeth throughout life without resorting to the use of a prosthesis" was adopted by the World Health Organization (WHO) as part of its oral health goals for developing countries. ${ }^{5,6}$ Subsequently, this concept has been included in the National Oral Health Policy of South Africa to ensure optimal oral health for all. However, inclusion and implementation at a practice level has been absent. ${ }^{2,3,7}$

Classically, patients having 10 pairs of occluding anterior and premolar teeth are considered to have SDAs. ${ }^{4,5}$ The clinical description of the SDA denotes the occluding posterior teeth as occluding units (OU), with one OU equalling two opposing premolars in occlusion and two OUs equalling two opposing molars in occlusion. ${ }^{5}$ Thus, the classic SDA comprises an intact anterior dentition and four symmetrically distributed posterior OUs. ${ }^{4,5}$ Other descriptions mentioned in the literature include posterior occluding pairs (POPs) of teeth with three to four POPs arranged symmetrically 
and five to six asymmetrically, or a posteriorly reduced dentition. ${ }^{4,5,8-20}$ These occlusal arrangements have been shown to be useful and have been accepted in some communities in terms of patients' ability to function, subjective satisfaction, and oral comfort with a positive impact on their oral health-related quality of life (OHRQoL). ${ }^{4,5,8-20}$

Both primary and secondary studies have indicated that the SDA as an alternative treatment approach is scientifically valid and has no harmful effects on the remaining dentition when prescribed appropriately. $4,5,8-10,12-15,17,21-32$ The broad findings of these studies state that: (1) 20 anterior and posterior occluding teeth (the classic SDA) are adequate for oral function, emphasizing the value of a functional dentition; (2) patient satisfaction increases with premolar occlusion, and adding occluding molars does not improve it any further; (3) occlusal stability and support are satisfactory with 3 to 4 POPs of symmetrically arranged and 5 to 6 POPs of asymmetrically arranged teeth; and (4) OHRQoL is directly proportional to 9 or more pairs of anterior and posterior occluding teeth.

Advantages of preserving a functional dentition with 20 teeth or 4 well-distributed OUs have been reported in the literature. ${ }^{4,5,16-18}$ Such an alternative to the normal 28 teeth when limitations such as cost and patient compliance and/or ability are a concern produces adequate function. The prosthodontic interventions normally used to replace molars include removable or fixed partial denture prostheses (RPDPs or FPDPs) and implant-supported prostheses. ${ }^{19,20}$ No difference regarding temporomandibular problems and no clinically significant differences in OHRQoL of patients who do not have molar teeth are reported. ${ }^{4,5,16,17}$ Indeed, the SDA is regarded as a rehabilitative or reconstructive alternative treatment option when its prescription is possible. ${ }^{19-20}$ More specifically, it can be considered an appropriate and relevant treatment strategy in developing countries, especially in a resource-constrained environment such as South Africa, for more effective management of the needs of the population. ${ }^{2,3}$

Correspondingly, problems related to the use of RPDPs that may mitigate against the extension of shortened arches to 28 teeth include the large number of those who find RPDPs unacceptable and choose not to wear them due to the limited retention and support, chewing incapacity, and unacceptable esthetics. ${ }^{4,5,8,10,18,21,22,33,34}$ Moreover, circumstances where patients would be advised against extension of a shortened arch include an increase in caries (especially root caries) and periodontal disease of remaining teeth, inconsistent reports of improvement in oral function when using distal-extension RPDPs, and the improvement in OHRQoL with RPDPs only when esthetics is a concern but to a lesser extent when chewing ability, speech, and comfort are important. 4,5,8,10,18,21,22,33,34

It is suggested that a rigorous overview related to the SDA will allow synthesis of the results from multiple systematic reviews (SRs) conducted in different parts of the world with slightly different inclusion criteria and resulting in different sample sizes but where the findings overlapped. ${ }^{35,36}$ Moreover, this SDA overview would facilitate identification of high-quality and reliable SRs on the topic, explore consistency of findings, create more evidence, and consequently strengthen the SDA evidence already collected and collated. ${ }^{35,36}$ Adopting such a rigorous methodology has advantages in that it allows summarizing of evidence already collected on the SDA, facilitating the process of translating this knowledge to clinical practice. ${ }^{35,37}$ This type of critical assessment of SRs related to the SDA concept has not been completed, thus it is a novel approach to doing secondary research. ${ }^{35,37}$

In addition, each included SR will be critically appraised using the AMSTAR tool, which assesses the methodologic quality of SRs (Fig 1). ${ }^{37}$ The AMSTAR checklist used for this study is an 11-question checklist with 4 responses (yes/no/cannot answer/ not applicable) and a score of 1 for each yes response (Fig 1). ${ }^{37}$ The ratings are grouped according to scores obtained into high (score of 8-11), medium (4-7), and low (0-3), with the responses following a rigorous explanation and interpretation of what constitutes a yes answer. ${ }^{37}$

The aim of this study was to identify high-quality SRs related to the SDA concept and its variants and to explore consistency of findings across reviews with specific reference to function, OHRQoL, and the various prosthodontic interventions that may be prescribed for the purpose of arch extension, when deemed appropriate.

\section{Materials and Methods}

\section{Protocol Development}

A protocol (Registration No: 15/2/9) was developed (not published) to include all aspects of an overview of SR, namely selection criteria, search strategy, selection methods using predetermined eligibility criteria, data collection, data extraction using a preformed data sheet, and AMSTAR tool to evaluate the methodologic quality of each included SR.

Ethical clearance for the primary studies that were included in each of the SRs used for this overview had to have been obtained from the respective institutions involved at that time. Written informed consent had 


\section{AMSTAR}

1. Was an a priori design provided?

The research question and inclusion criteria should be established before the review is conducted.

Note: Need to refer to a protocol, ethics approval, or predetermined/a priori published research objectives to score a "yes."
$\square$ Yes
$\square$ Can't answer
$\square$ No
Not applicable

2. Was there duplicate study selection and data extraction? There should be at least two independent data extractors, and a consensus procedure for disagreements should be in place.
$\square$ Yes
$\square$ Can't answer
$\square$ No
$\square$ Not applicable

3. Was a comprehensive literature search performed? At least two electronic sources should be searched. The report must include years and databases used (eg, Central, EMBASE, MEDLINE). Key words and/or MeSH terms must be stated, and where feasible, the search strategy should be provided. All searches should be supplemented by consulting current content, reviews, textbooks, specialized registers, or experts in the particular field of study, and by reviewing the references in the studies found.
$\square$ Yes
$\square$ Can't answer
$\square$ No
$\square$ Not applicable

4. Was the status of publication (ie, grey literature) used as an inclusion criterion?

The authors should state that they searched for reports regardless of publication type. The authors should state whether or not they excluded any reports (from the systematic review), based on publication status, language, etc.
$\square$ Yes
$\square$ Can't answer
$\square \mathrm{No}$
$\square$ Not applicable

5. Was a list of studies (included and excluded) provided? A list of included and excluded studies should be provided.
$\square$ Yes
$\square$ Can't answer
$\square \mathrm{No}$
$\square$ Not applicable

6. Were the characteristics of the included studies provided? In an aggregated form such as a table, data from the original studies should be provided on the participants, interventions, and outcomes. The ranges of characteristics in all the studies analyzed (eg, age, race, sex, relevant socioeconomic data, disease status, duration, severity, or other diseases) should be reported.
$\square$ Yes
$\square$ Can't answer
$\square$ No
$\square$ Not applicable

7. Was the scientific quality of the included studies assessed and documented?

A priori methods of assessment should be provided (eg, for effectiveness studies if the authors chose to include only randomized, double-blinded, placebo-controlled studies, or allocation concealment as inclusion criteria); for other types of studies alternative items will be relevant.
$\square$ Yes
$\square$ Can't answer

$\square$ No

$\square$ Not applicable

8. Was the scientific quality of the included studies used appropriately in formulating conclusions?

The results of the methodological rigor and scientific quality should be considered in the analysis and the conclusions of the review and explicitly stated in formulating recommendations.
$\square$ Yes
$\square$ Can't answer
$\square$ No
$\square$ Not applicable

9. Were the methods used to combine the findings of studies appropriate?

For the pooled results, a test should be done to ensure the studies were combinable, to assess their homogeneity (ie, chi-square test for homogeneity, I2). If heterogeneity exists, a random effects model should be used and/or the clinical appropriateness of combining should be taken into consideration (ie, is it sensible to combine?).
$\square$ Yes
$\square$ Can't answer
$\square$ No
$\square$ Not applicable

10. Was the likelihood of publication bias assessed?

An assessment of publication bias should include a combination of graphical aids (eg, funnel plot) and/or statistical tests (eg, Egger regression test, Hedges-Olken).
$\square$ Yes
$\square$ Can't answer
$\square$ No
$\square$ Not applicable

11. Was the conflict of interest included?

Potential sources of support should be clearly acknowledged in both the systematic review and the included studies.

$\square$ Yes

$\square$ No

Fig 1 The AMSTAR tool, a measurement tool used to assess the methodologic quality of systematic reviews. ${ }^{37}$

also been obtained from the participants in the primary studies according to the Declaration of Helsinki. ${ }^{38}$

\section{Criteria for Considering Studies for This Overview}

All systematic reviews making reference to SDAs, including those describing different patterns of tooth arrangements and discussing interventions used for SDAs, were included. Men and women aged 18 years and older and having different SDAs and/or posterior reduced dental arches were included. Primary and secondary outcomes were prespecified. Primary outcomes were subjective or investigator- or patient-reported outcomes, including outcomes focusing on, for 
example, function, patient satisfaction, and OHRQoL in patients with SDAs or any related tooth arrangements. Secondary outcomes were survival of teeth in patients with SDAs, arrangement and location of teeth (patterns of tooth loss), and survival of prosthodontic intervention (RPDPs, FPDPs, and implant-retained prostheses) used to treat SDAs.

SRs related to SDAs (including those describing the location of teeth for SDAs) and studies that discuss prosthodontic interventions used for SDA patients were included. Primary and secondary research studies on animals that did not meet the inclusion criteria were excluded from this review.

A computerized search was conducted for all SRs for the period of January 2000 to August 2016 to identify literature related to the SDA, including studies using the SDA as a treatment strategy for partially dentate adult patients within the following databases: MEDLINE, CINAHL, the Cochrane Central Register of Controlled Trials (CENTRAL) of the Cochrane Library, Science Direct, Science Journals, Scopus, Dentistry and Oral Science Source (DOSS), Springerlink, and Wiley. ${ }^{35,36}$ Further hand searching was also conducted from reference lists of retrieved studies (PEARLing searches).

Key terms were combined using Boolean operators, and search strategies for each database and these were developed using their specific functions. A broad search strategy was used and it focused on types of reviews related to patients with SDAs: (shortened dental arch OR shortened dental arches) AND (literature reviews OR reviews OR systematic review OR meta-analysis OR meta-analyses) AND (2000/01/01-2016/08/31).

Databases were initially searched for SRs published in English from January 2000 to December 2015. Another search was conducted on August 2016. The limits included human studies, adult patients, and SRs.

\section{Selection Methods}

An eligibility form compiled from the inclusion criteria was used by the review authors and a research assistant to independently screen and include potentially relevant studies. ${ }^{35}$ Reasons for inclusion were reported. Full-text articles were retrieved, and data extraction was completed by the principal researcher and a research assistant on study designs, methods, participants, interventions, outcomes, and conclusions from each SR using a specially designed prepiloted data extraction form. ${ }^{36}$ Disagreements regarding data extraction were resolved by discussion among all reviewers.

The primary author and a research assistant independently completed the AMSTAR checklist to critically assess the methodologic quality of SRs (Fig 1). ${ }^{37}$

\section{Qualitative Analysis}

A qualitative discussion related to the primary and secondary outcomes stipulated for this overview from the data extracted from each SR (Table 1). In addition, the AMSTAR checklist was completed to assess the quality of each included SR and the scores were calculated using the online system where a yes answer equalled a score of 1 and any other response equalled a score of $0 .{ }^{37}$ Results of the AMSTAR evaluation are summarized in Tables 2 and 3. Observer agreement scores were calculated, and disagreements were again resolved by discussion among the research assistant and review authors.

\section{Data Synthesis and Management}

This process included analyzing all Cochrane and non-Cochrane SRs, collating and reporting the results separately for the outcomes-namely, the effects of the SDA on patient satisfaction, function, OHRQoL, and arrangement of teeth. In addition, characteristics of each included SR were collated and comparisons between SRs reported using tables and by discussion. The results also include a report on the methodologic quality of each included SR according to the AMSTAR checklist and summarized in the tables.

\section{Results}

A comprehensive search generated a combined total of 45 articles and reviews related to SDAs (Fig 2). Duplicates $(n=21)$ of SDA articles obtained from the different search engines were excluded, leaving only review articles $(n=24)$. These articles included other types of nonsystematic reviews, after exclusion of which only five SRs were left. An additional four were found through hand searching for a final sample size of nine SRs, as shown in Fig 2. No SRs were found earlier than 2007; the final 9 SRs were from the period of 2007 to 2016, which included a total of 228 articles (Table 2). ${ }^{13,23-31}$

\section{Study Characteristics}

The key features of the included SRs are summarized and reported in Table 2. These are recorded by author, year, location where SR was conducted, aims and/ or objectives, outcomes, conclusions, and the findings related to the SDA (Table 2). While SRs related to SDAs were conducted in eight different countries, each research group investigated different but related aspects of SDA research (Tables 2 and 3 ).

Design. The nine included SRs could be broadly grouped according to their included study designs 
Table 1 Characteristics of Included Systematic Reviews

\begin{tabular}{|c|c|c|c|c|c|}
\hline Author(s) & $\mathrm{n}$ & Objectives & Outcomes & Conclusions & SDA \\
\hline Abt et $\mathrm{al}^{29}$ & 21 & $\begin{array}{l}\text { Assess effects of different } \\
\text { prostheses for a partially absent } \\
\text { dentition }\end{array}$ & $\begin{array}{l}\text { Primary: long-term } \\
\text { success } \\
\text { Secondary: function, } \\
\text { morbidity, PS }\end{array}$ & $\begin{array}{l}\text { Insufficient evidence to say } \\
\text { one intervention better than } \\
\text { others; not all outcomes } \\
\text { were reported }\end{array}$ & \\
\hline Faggion $^{26}$ & 9 & $\begin{array}{l}\text { Systematically assess outcomes } \\
\text { from nontreatment and treatment } \\
\text { approaches for SDA cases; } \\
\text { assess effectiveness of restorative } \\
\text { approaches for SDAs; assess quality } \\
\text { of retrieved evidence (using GRADE) }\end{array}$ & $\begin{array}{l}\text { Qualitative: QoL, } \\
\text { function, esthetics } \\
\text { Quantitative: } \\
\text { temporomandibular } \\
\text { disorder, occlusal } \\
\text { problems, tooth loss }\end{array}$ & $\begin{array}{l}\text { No difference between the } \\
\text { two approaches; two studies } \\
\text { showed treatment of SDAs } \\
\text { with FPDP greater benefit } \\
\text { compared to RPDP }\end{array}$ & Positive \\
\hline Fueki et $\mathrm{a}^{28}$ & 21 & $\begin{array}{l}\text { To review literature for effect of } \\
\text { prosthetic restorations on SDA } \\
\text { patients: whether RPDPs for distal } \\
\text { extensions increases function/PS/ } \\
\text { OHRQoL vs FPDPs; advantages and } \\
\text { disadvantages of treatment with } \\
\text { RPDP over FPDP and SDA }\end{array}$ & $\begin{array}{l}\text { Chewing; PS, QoL, } \\
\text { function, periodontal } \\
\text { problems, survival of } \\
\text { treatment }\end{array}$ & $\begin{array}{l}\text { RCTS conducted in Europe } \\
\text { not generalizable to Japan } \\
\text { due to socioeconomic } \\
\text { and/or healthcare system } \\
\text { differences }\end{array}$ & Positive \\
\hline Gerritsen et al ${ }^{25}$ & 35 & $\begin{array}{l}\text { Analyze relationship between } \\
\text { number/location of missing teeth } \\
\text { and OHRQoL: Is TL associated with } \\
\text { impaired OHRQoL? What is the role } \\
\text { of location and distribution of tooth } \\
\text { location? }\end{array}$ & $\begin{array}{l}\text { Primary: TL associated } \\
\text { with impairment of } \\
\text { OHRQoL } \\
\text { Secondary: location } \\
\text { and distribution of teeth } \\
\text { affect OHRQoL }\end{array}$ & $\begin{array}{l}\text { Strong evidence that TL is } \\
\text { associated with impairment } \\
\text { of OHRQoL and location of } \\
\text { TL affects severity }\end{array}$ & Positive \\
\hline $\begin{array}{l}\text { Gotfredsen and } \\
\text { Walls }^{13}\end{array}$ & 83 & $\begin{array}{l}\text { Evaluate relationship between } \\
\text { dentition and oral function }\end{array}$ & $\begin{array}{l}\text { Masticatory function, } \\
\text { esthetics, PS, occlusal } \\
\text { support/stability, other } \\
\text { functions (tactile/ } \\
\text { phonetics/taste) }\end{array}$ & $\begin{array}{l}\text { Few studies with high level } \\
\text { of evidence; low evidence: } \\
\text { masticatory efficiency } \\
\text { decrease with TL but 9-10 } \\
\text { OUs ensures functioning/ } \\
\text { stability; dietary intake and } \\
\text { OHRQoL unchanged with } \\
\text { 9-10 OUs }\end{array}$ & Positive \\
\hline Khan et $\mathrm{a}^{30}$ & 21 & $\begin{array}{l}\text { Compare SDA and CDA; compare } \\
\text { differences between interventions } \\
\text { (FPDs/RPDs) used to extend } \\
\text { SDAs; determine PS with these } \\
\text { interventions; determine functional } \\
\text { outcomes with different interventions }\end{array}$ & $\begin{array}{l}\text { Primary: functional } \\
\text { outcomes, survival of } \\
\text { intervention } \\
\text { Secondary: PS, negative } \\
\text { effects }\end{array}$ & $\begin{array}{l}\text { SDA as a treatment option is } \\
\text { encouraging (function/PS/ } \\
\text { costs); RCTs conducted in } \\
\text { Europe not generalizable to } \\
\text { South Africa due to cultural } \\
\text { differences }\end{array}$ & Positive \\
\hline $\begin{array}{l}\text { Shahmiri and } \\
\text { Atieh }^{23}\end{array}$ & 9 & $\begin{array}{l}\text { Evaluate the use of implant- } \\
\text { tooth-borne RPDPs in prosthetic } \\
\text { rehabilitation of Kennedy Class I } \\
\text { partially edentulous arches; evaluate } \\
\text { existing evidence to determine } \\
\text { whether implant-supported RPDs } \\
\text { provided better performance } \\
\text { compared to other treatments }\end{array}$ & $\begin{array}{l}\text { PS, masticatory } \\
\text { efficiency, bone loss, } \\
\text { prosthetic maintenance, } \\
\text { soft and hard tissue } \\
\text { response }\end{array}$ & $\begin{array}{l}\text { Improvement in function, } \\
\text { esthetics, and stability } \\
\text { has been demonstrated } \\
\text { in all studies with minimal } \\
\text { prosthetic care, but RCTs are } \\
\text { needed to provide evidence } \\
\text { that will validate use of these } \\
\text { treatment modalities }\end{array}$ & Positive \\
\hline Liang et $\mathrm{al}^{31}$ & 8 & $\begin{array}{l}\text { To synthesize available knowledge } \\
\text { about effects of distal extension } \\
\text { RPDPs on masticatory performance } \\
\text { of subjects with moderate or } \\
\text { extreme SDA }\end{array}$ & $\begin{array}{l}\text { Comminuting, chewing, } \\
\text { mixing ability, occlusal } \\
\text { force }\end{array}$ & $\begin{array}{l}\text { Patients with extreme SDA } \\
\text { had } 30 \%-40 \% \text { reduction in } \\
\text { masticatory performance; } \\
\text { distal extension RPDPs } \\
\text { partially compensated } \\
\text { for performance }(50 \%) ; \\
\text { more false teeth on } \\
\text { RPDPs resulted in better } \\
\text { performance }\end{array}$ & $\begin{array}{l}\text { Positive } \\
\text { toward SDA, } \\
\text { not the } \\
\text { extreme SDA }\end{array}$ \\
\hline Zhang et $\mathrm{al}^{24}$ & 21 & $\begin{array}{l}\text { To assess oral health and } \\
\text { prosthodontic conditions of } \\
\text { Chinese adults over time: review } \\
\text { DMFT and number and location of } \\
\text { teeth in adults; consider need for } \\
\text { prosthodontic appliances }\end{array}$ & $\begin{array}{l}\text { Mean DMFT values, } \\
\text { components of DMFT, } \\
\text { number of teeth/roots/ } \\
\text { occluding teeth }\end{array}$ & $\begin{array}{l}\text { Insufficient information } \\
\text { to answer objectives as } \\
\text { outcomes }\end{array}$ & \\
\hline
\end{tabular}

PS = patient satisfaction; SDA = shortened dental arch; QoL = quality of life; FPDP = fixed partial dental prosthesis; RPDP = removable partial denture prosthesis; $\mathrm{OHRQoL}=$ oral health-related quality of life; $\mathrm{TL}=$ tooth loss; $\mathrm{OU}=$ occlusal unit; $\mathrm{CDA}=$ complete dental arch; FPD = fixed partial denture; $\mathrm{RPD}=$ removable partial denture; RCT = randomized controlled trial; DMFT = decayed, missing, and filled teeth . 


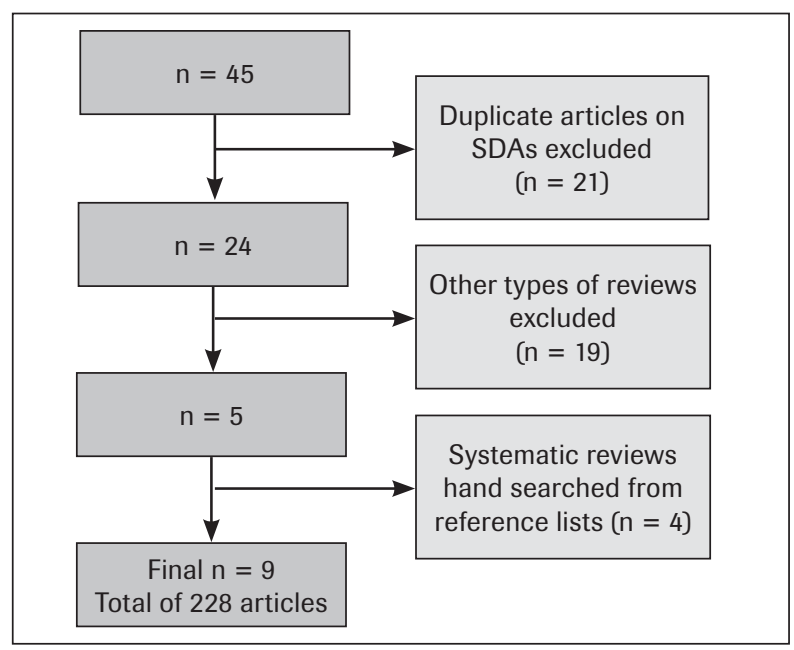

Fig 2 Flow chart of included systematic reviews (electronic and PEARLing searches).

into (1) those where only clinical trials were included and (2) those that included a range of designs.

For group 1, two of the SRs ${ }^{29,30}$ used only RCTs and/ or nonrandomized controlled clinical trials in their analyses. Both used the Cochrane format to conduct the SR, and both completed quality assessments of the evidence for any risk of bias using the Cochrane Risk of Bias tool. ${ }^{29,30,36}$ SRs that include only clinical trials are considered to be of a high standard as the primary studies follow a rigorous methodology, and the SRs follow an equally strict methodology by including the assessment of the risk of bias of each included study. ${ }^{36}$

For group 2, six of the included SRs ${ }^{23-28,31}$ each included a range of designs (cross-sectional, cohort, and case reports) and indicated that they did not exclude any studies based on design. Gotfredsen and Walls, ${ }^{13}$ while including a mixed range of designs, nevertheless excluded other study types, such as case reports, expert opinions, animal studies, and technical descriptions.

Thus, it can be said that the ideal for an SR that includes only clinical trials was not strictly followed by several of the SRs included in this overview. . $^{13,23-31}$

Research Questions. While the research questions of the included SRs differed, they were all still related to the SDA. The articles reviewed in the SRs included in this overview were mostly the same. The research questions for the SRs related to interventions used oral function, impact on OHRQoL, location, and tooth arrangements with one epidemiologic study determining the state of teeth for a specific Chinese community. ${ }^{13,23-31}$ Not surprisingly, the more specific the research question, the fewer articles were included in the analysis. ${ }^{23,26,27,31}$ For example, Gotfredsen
Table 2 AMSTAR Scores for Included Systematic Reviews

\begin{tabular}{lclcc}
\hline Author & Year & \multicolumn{1}{c}{ Country } & $\begin{array}{c}\text { AMSTAR } \\
\text { Total/11 }\end{array}$ & Grade \\
\hline Abt et al $^{29}$ & 2012 & USA & 10 & $\mathrm{H}$ \\
Faggion $^{26}$ & 2011 & Germany & 8 & $\mathrm{H}$ \\
Fueki et al $^{28}$ & 2011 & Japan & 5 & $\mathrm{M}$ \\
\hline Gerritsen et al $^{25}$ & 2010 & Netherlands & 8 & $\mathrm{H}$ \\
\hline $\begin{array}{l}\text { Gotfredsen and } \\
\text { Walls }^{13}\end{array}$ & 2007 & Denmark & 6 & $\mathrm{M}$ \\
Khan et al $^{30}$ & 2014 & South Africa & 9 & $\mathrm{H}$ \\
\hline $\begin{array}{l}\text { Shahmiri and } \\
\text { Atieh }^{23}\end{array}$ & 2007 & New Zealand & 8 & $\mathrm{H}$ \\
\hline Liang et al & & & & \\
\hline Zhang et al & & & & \\
\hline
\end{tabular}

$\mathrm{H}=$ high score (8-11); $\mathrm{M}=$ medium score (4-7); $\mathrm{L}=$ low score (0-3).

and Walls ${ }^{13}$ used a broad research question; thus, more articles were included in their study (Table 2).

Outcomes of Each SR. Study outcomes should be prespecified as primary or secondary when conducting an SR. However, outcomes were specified as primary and/or secondary in only three out of the nine included SRs. ${ }^{25,29,30}$ Aside from the epidemiologic study, the study outcomes focused mainly on function, esthetics, patient satisfaction, and QoL (Table 2).

When comparing the primary outcomes stipulated for the present overview to those of the included SRs, it was noted (Table 2) that most of the included SRs ( $\mathrm{n}=$ 8) provided evidence for at least one primary outcome that was also stipulated for this overview, ${ }^{13,23,25-31}$ with seven SRs investigating two or more of these primary outcomes. ${ }^{13,23,25,28-31}$ Specifically, seven SRs assessed oral function ${ }^{13,23,26-31}$ and five assessed patient satisfaction ${ }^{13,25,28-30}$ and OHRQ.L.. ${ }^{3,25,28,30,31}$

Regarding secondary outcomes, four SRs looked at tooth loss, ${ }^{13,24,26,30}$ four investigated survival of intervention, ${ }^{23,28-30}$ and two looked at number, arrangement, and location of teeth. 24,25

Conclusions of Each SR. Abt et al ${ }^{29}$ stated that there was not enough evidence to definitively conclude that one intervention is better than the other, thus the research question was not answered (Table 2). Khan et $\mathrm{al}^{30}$ specified that the SDA as a treatment option was encouraging as regards function, patient satisfaction, and cost, even though sufficient RCTs with acceptable rigor have not been conducted (Table 2). Khan et $\mathrm{al}^{30}$ and Fueki et $\mathrm{al}^{28}$ concluded that the results from certain regions may not be generalizable to the rest of the world due to cultural and/or socioeconomic differences (Table 2). It was also mentioned 
Table 3 Responses of Systematic Reviews to the AMSTAR Questions

\begin{tabular}{|c|c|c|c|c|c|c|c|c|c|c|c|}
\hline \multirow{2}{*}{\multicolumn{2}{|c|}{ AMSTAR item }} & \multirow[b]{2}{*}{$\begin{array}{l}\text { Reviews } \\
\text { meeting } \\
\text { criteria } \\
\text { (n) }\end{array}$} & \multicolumn{9}{|c|}{ Systematic review researchers } \\
\hline & & & \multirow{2}{*}{$\begin{array}{c}\begin{array}{c}\text { Abt } \\
\text { et } \mathrm{al}^{29}\end{array} \\
\mathrm{Y}\end{array}$} & \multirow{2}{*}{$\frac{\text { Faggion }^{26}}{\mathrm{Y}}$} & \multirow{2}{*}{$\begin{array}{c}\begin{array}{c}\text { Fueki } \\
\text { et } \mathrm{al}^{28}\end{array} \\
\mathrm{Y}\end{array}$} & \multirow{2}{*}{$\begin{array}{c}\begin{array}{c}\text { Gerritsen } \\
\text { et }^{2 l^{25}}\end{array} \\
\mathrm{Y}\end{array}$} & \multirow{2}{*}{$\begin{array}{c}\begin{array}{c}\text { Gotfredsen } \\
\text { and Walls }{ }^{13}\end{array} \\
\mathrm{Y}\end{array}$} & \multirow{2}{*}{$\begin{array}{c}\begin{array}{c}\text { Khan } \\
\text { et } \mathrm{al}^{30}\end{array} \\
\mathrm{Y}\end{array}$} & \multirow{2}{*}{$\begin{array}{c}\begin{array}{c}\text { Liang } \\
\text { et }^{31}\end{array} \\
Y \mathrm{Y}\end{array}$} & \multirow{2}{*}{$\begin{array}{c}\begin{array}{c}\text { Shahmiri } \\
\text { and } \\
\text { Atieh }^{23}\end{array} \\
Y\end{array}$} & \multirow{2}{*}{$\begin{array}{c}\begin{array}{c}\text { Zhang } \\
\text { et } \mathrm{al}^{24}\end{array} \\
\mathrm{Y}\end{array}$} \\
\hline 1 & Was an a priori design provided? & 9 & & & & & & & & & \\
\hline 2 & $\begin{array}{l}\text { Was there duplicate study } \\
\text { selection and data extraction? }\end{array}$ & 7 & $\mathrm{Y}$ & $\mathrm{N}$ & $\mathrm{Y}$ & Y & $\mathrm{N}$ & $\mathrm{Y}$ & $\mathrm{Y}$ & Y & $\mathrm{Y}$ \\
\hline 3 & $\begin{array}{l}\text { Was a comprehensive literature } \\
\text { search performed? }\end{array}$ & 8 & $\mathrm{Y}$ & $\mathrm{Y}$ & $\mathrm{Y}$ & $\mathrm{Y}$ & $\mathrm{Y}$ & $\mathrm{Y}$ & $\mathrm{Y}$ & $\mathrm{Y}$ & $\mathrm{N}$ \\
\hline 4 & $\begin{array}{l}\text { Was the status of publication } \\
\text { used as inclusion criteria? }\end{array}$ & 6 & $\mathrm{Y}$ & $\mathrm{Y}$ & $\mathrm{N}$ & Y & $\mathrm{N}$ & $\mathrm{Y}$ & $\mathrm{N}$ & $\mathrm{Y}$ & $\mathrm{Y}$ \\
\hline 5 & $\begin{array}{l}\text { Was a list of included and } \\
\text { excluded studies provided? }\end{array}$ & 8 & $\mathrm{Y}$ & $\mathrm{Y}$ & $\mathrm{N}$ & $\mathrm{Y}$ & $\mathrm{Y}$ & $\mathrm{Y}$ & $\mathrm{Y}$ & $\mathrm{Y}$ & $\mathrm{Y}$ \\
\hline 6 & $\begin{array}{l}\text { Were the characteristics of } \\
\text { included studies provided? }\end{array}$ & 9 & $\mathrm{Y}$ & $\mathrm{Y}$ & $\mathrm{Y}$ & $\mathrm{Y}$ & $\mathrm{Y}$ & $\mathrm{Y}$ & $\mathrm{Y}$ & $\mathrm{Y}$ & $\mathrm{Y}$ \\
\hline 7 & $\begin{array}{l}\text { Was scientific quality of included } \\
\text { studies assessed and reported? }\end{array}$ & 5 & $Y$ & $Y$ & $\mathrm{~N}$ & $\mathrm{~N}$ & $Y$ & $Y$ & $\mathrm{~N}$ & $Y$ & $\mathrm{~N}$ \\
\hline 8 & $\begin{array}{l}\text { Was scientific quality of } \\
\text { studies used appropriately in } \\
\text { formulating conclusions? }\end{array}$ & 5 & $Y$ & $\mathrm{Y}$ & $\mathrm{N}$ & $\mathrm{N}$ & $Y$ & $Y$ & $\mathrm{~N}$ & $\mathrm{Y}$ & $\mathrm{N}$ \\
\hline 9 & $\begin{array}{l}\text { Were the methods used to } \\
\text { combine findings appropriate? }\end{array}$ & 2 & $Y$ & $\mathrm{~N}$ & $\mathrm{~N}$ & $\mathrm{Y}$ & $\mathrm{N}$ & $\mathrm{N}$ & $\mathrm{N}$ & $\mathrm{N}$ & $\mathrm{N}$ \\
\hline 10 & $\begin{array}{l}\text { Was the likelihood of publication } \\
\text { bias assessed? }\end{array}$ & 0 & $\mathrm{~N}$ & $\mathrm{~N}$ & $\mathrm{~N}$ & $\mathrm{~N}$ & $\mathrm{~N}$ & $\mathrm{~N}$ & $\mathrm{~N}$ & $\mathrm{~N}$ & $\mathrm{~N}$ \\
\hline 11 & $\begin{array}{l}\text { Was the conflict of interest } \\
\text { included? }\end{array}$ & 6 & $Y$ & $\mathrm{Y}$ & $\mathrm{Y}$ & $Y$ & $\mathrm{~N}$ & $\mathrm{Y}$ & $\mathrm{Y}$ & $\mathrm{N}$ & $\mathrm{N}$ \\
\hline AMS & TAR score & & 10 & 8 & 5 & 8 & 6 & 9 & 6 & 8 & 5 \\
\hline
\end{tabular}

$\mathrm{Y}=$ yes (score of 1$) ; \mathrm{N}=$ no (score of 0 ).

that primary studies with a rigorous study design were visibly absent, and it was thus recommended that more RCTs following a strict protocol should be conducted (Table 2).

With specific reference to the SDA, seven of the nine SRs supported and recommended that the SDA concept be included as a viable treatment option when appropriate (Table 2).

\section{Quality of Evidence}

Quality assessments of studies, whether primary or secondary research, add reliability and allow the merging of study outcomes, and are thus always recommended. ${ }^{35-37}$ The SRs conducted by Faggion ${ }^{26,27}$ also assessed the quality of evidence of the included primary studies using the Grading of Recommendations Assessment, Development and Evaluation (GRADE) approach, as did Khan et al. ${ }^{30}$ These studies evaluated the evidence and strength of recommendations for clinical interventions of each included clinical trial in the respective SR. ${ }^{26,27}$ Khan et $\mathrm{a}^{30}$ also assessed the quality of the evidence using the risk of bias tool for all included clinical trials, as was mentioned previously.
The quality of the evidence for the present overview of SRs was determined using an AMSTAR checklist by assessing the methodologic rigor of each included study. Each of the nine SRs had a high $(n=5)^{24-27,29,30}$ or a medium $(n=4)^{13,23,28,31}$ AMSTAR score (Table 3 and Fig 1).

Table 3 highlights the responses for each of the 11 AMSTAR questions, indicating that all the SRs provided the design and some characteristics of the studies included. ${ }^{13,23-31,37}$ None of the included SRs, however, assessed publication bias, which is normally indicated by funnel plots or statistical tests such as Egger regression tests; thus, a score of zero was recorded for this question (Table 3). ${ }^{13,23-31,36}$ The AMSTAR evaluation showed that some of the included SR researchers had not carried out a quality assessment of the included primary studies, so drawing definitive conclusions was not possible. ${ }^{37}$ Only six of the nine SRs reported a conflict of interest statement (Table 3), an item that has become mandatory when submitting articles to scientific journals. ${ }^{37}$

Disagreements Between Researchers Related to the AMSTAR Assessment. The 11-question AMSTAR checklist was completed independently by the primary author and a research assistant (Fig 1). 
On completion, disagreements between the principal author and the research assistant were observed. The Pearson correlation coefficient at 0.494 indicated that the differences were not significant $(P=.177)$. The disagreements were discussed, and when consensus could not be reached the other review authors were brought in to resolve the disagreement.

For the SRs by Abt et $\mathrm{al}^{29}$ and Liang, ${ }^{31}$ researchers were in total agreement in their AMSTAR scoring. Differences in scoring were found most often with questions 2, 8, and 9 (Fig 1) and for two or more of the SRs. ${ }^{13,24,25,28,30,35}$ These differences could easily be linked to the reporting of how the study was conducted. With questions 3 and 6, authors reached a consensus that even if not all characteristics of a study (eg, year, databases searched, participant demographics) were included (Fig 1), the AMSTAR score for the SR would still be recorded as yes. ${ }^{35}$

\section{Discussion}

Although some variations were observed between the different SRs with respect to research questions, outcomes, and conclusions, the evidence, once collated and summarized, can be regarded as reliable. For overviews, however, results are hampered by the fact that review protocols and outcome measures of the component SRs cannot be assumed to have been consistent. For this reason, the findings of the present overview are reported in the form of a narrative. ${ }^{36}$

This overview covered a range of aspects of SDA research in the form of SRs. ${ }^{13,23-31}$ Among these were aspects related to tooth arrangements and their effect on QoL and OHRQoL, epidemiologic studies determining the patterns of tooth loss among older communities, and the different interventions used to extend SDAs. ${ }^{13,23-31}$

The results of the present overview showed that a number of different interventions are variously employed for SDA patients ranging from RPDPs, FPDPs, resin-bonded bridges, and implant-supported prostheses. It was also found that an SDA with 9 to 10 occluding units adequately satisfies the oral functional needs of many patients. ${ }^{13,23-31}$ Studies have also indicated the negative effect an RPDP (especially distal extension mandibular dentures) may have on patients. ${ }^{39,40}$ The positive outcomes with implant-supported procedures that may be considered ideal are hardly available to those in already resource-constrained developing countries and disadvantaged communities. ${ }^{23}$

These conclusions support the oral functionality of the SDA concept and are in line with other primary and secondary research studies related to function, indicating that restoration of a shortened arch to completeness may, in certain clinical conditions, be considered overtreatment. $4,5,8-15,17-20,22-34$ It has also been reported that QoL is not negatively affected by an SDA management approach, although it may be affected with an extreme SDA.13,17,1921,25,28,30,39-42 The SDA approach further emphasizes how socioeconomic constraints and issues of poor access for care experienced by patients can be addressed. The evidence gathered through the appraisal of the included SRs by means of a reliable tool indicates support for a noninterventionist approach in certain cases of reduced posterior occlusions, $4,5,8-13,15,17-20,22-31,33,35,37,39-42$ benefitting underprivileged communities.

\section{Quality of the Evidence}

Though the quality of the evidence as assessed using the AMSTAR tool was acceptable (SRs had a medium or high score), the quality of the component primary studies making up the various SRs had not been assessed for most of the clinical trials, either by performing Cochrane risk of bias or using the GRADE analysis. ${ }^{36,43}$ In addition, publication bias was not assessed for any of the included SRs. ${ }^{36}$ However, this did not affect the quality of the SRs given the generally high AMSTAR scores. ${ }^{36,43}$ It would be useful, though, to ensure that quality assessments are completed at primary and secondary research levels.

\section{Implications for Practice}

It is recommended that the continuing disjuncture between the evidence for the positive role of the SDA concept and dental clinical education, continuing education, and clinical implementation be addressed. Including the SDA concept in undergraduate clinical education would be an important step in adjusting the longstanding clinical paradigm of tooth replacement to a complete 28-tooth arch. The benefit of the SDA approach in disadvantaged communities is substantial. Better translation of the SDA concept into clinical practice should be pursued. Barriers known to hinder this critical phase need to be highlighted; the evidence gathered over the last 35 years must be shared with decision makers and clinical teachers.

\section{Implications for Research}

The reasons for the failure in knowledge translation for concepts such as the SDA, which has been extensively researched and corroborated, need to be explored further. ${ }^{44}$ Specifically, the acceptance of the SDA amongst communities who have been made aware of its benefits should be researched. 


\section{Conclusions}

The research questions, types of studies, and study outcomes of each included SR varied, which meant that the conclusions of each were somewhat different from the others. Nevertheless, most of the SRs $(n=7)$ emphasized the significance of the SDA concept as a functionally satisfactory approach to managing certain groups of partially dentate patients. According to the AMSTAR evaluation, the methodologies of the included SRs were of a high standard and most were of good quality. Reliance on their results would be acceptable. ${ }^{37,43}$

\section{Acknowledgments}

The present research was presented at the International Association of Dental Research (South Africa Division) in Pretoria, South Africa, in September 2015. The authors thank Dr Q. Isaacs for her invaluable assistance and knowledge related to this research, especially the assessment of each SR according to AMSTAR. The authors reported no conflicts of interest related to this study.

\section{References}

1. White HD, Van de Werf FJ. Thrombolysis for acute myocardial infarction. Circulation 1998:97:1632-1646.

2. Khan BS, Omar R, Chikte UM. Perceptions regarding the shortened dental arch among dental practitioners in the Western Cape Province, South Africa. SADJ 2012;67:60-68.

3. Khan BS, Chikte UM, Omar R. From classroom teaching to clinical practice: Experiences of senior dental students regarding the shortened dental arch concept. J Dent Educ 2014;78:906-913.

4. Käyser AF. Shortened dental arches and oral function. J Oral Rehabil 1981:8:457-462

5. Käyser AF. Shortened dental arch: A therapeutic concept in reduced dentitions and certain high-risk groups. Int Periodontics Restorative Dent 1989;9:426-449.

6. World Health Organization. A review of current recommendations for the organization and administration of Community Oral Health services in Northern and Western Europe: Report on a WHO Workshop: Oslo 24-28 May 1982. Copenhagen: WHO Regional Office for Europe, 1982.

7. Department of National Health and Population Development National Policy for Oral Health in South Africa, Act 116. National Policy for Oral Health, 1990.

8. Allen PF, Witter DF, Wilson NH, Käyser AF. Shortened dental arch therapy: Views of consultants in restorative dentistry in the United Kingdom. J Oral Rehabil 1996;23:481-485.

9. Witter DJ, van Elteren P, Käyser AF. Signs and symptoms of mandibular dysfunction in shortened dental arches. J Ora Rehabil 1988;15:413-420.

10. Witter DJ, van Elteren P, Käyser AF, van Rossum MJ. The effect of removable partial dentures on oral function in shortened dental arches. J Oral Rehabil 1989;16:27-33.

11. Carlsson GE. Some dogmas related to prosthodontics, temporomandibular disorders and occlusion. Acta Odontol Scand 2010;68:313-322.
12. Creugers NH, Witter DJ, Van 't Spijker A, Gerritsen AE, Kreulen $\mathrm{CM}$. Occlusion and temporomandibular function among subjects with mandibular distal extension removable partial dentures [epub 5 July 2010]. Int J Dent 2010;2010:807850.

13. Gotfredsen K, Walls AW. What dentition assures oral function? Clin Oral Implants Res 2007;18(Suppl 3):34-45.

14. Zhang Q, Witter DJ, Bronkhorst EM, Creugers NH. Chewing ability in an urban and rural population over 40 years in Shandong Province, China. Clin Oral Invest 2013;17:1425-1435.

15. Elias AC, Sheiham A. The relationship between satisfaction with mouth and number and position of teeth. J Oral Rehabil 1998;25:649-661.

16. Owen CP. Appropriatech: Prosthodontics for the many, not just for the few. Int J Prosthodont 2004;17:261-262.

17. Wolfart S, Heydecke G, Luthardt RG, et al. Effects of prosthetic treatment for shortened dental arches on oral health-related quality of life, self-reports of pain and jaw disability: Results from the pilot-phase of a randomized multicentre trial. J Oral Rehabil 2005;32:815-822.

18. Fejerskov $\mathrm{O}$, Escobar G, Jøssing M, Baelum V. A functional natural dentition for all-and for life? The oral healthcare system needs revision. J Oral Rehabil 2013;40:707-722.

19. De Sa e Frias V, Toothaker R, Wright RF. Shortened dental arch: A review of current treatment concepts. J Prosthodont 2004:13:104-110.

20. Armellini DB, von Fraunhofer JA. The shortened dental arch: A review of the literature. J Prosthet Dent 2004;92:531-535.

21. Käyser AF, Witter DJ, Spanauf AJ. Overtreatment with removable partial dentures in shortened dental arches. Aust Dent $J$ 1987:32:178-182.

22. Thomason JM, Moynihan PJ, Steen N, Jepson NJ. Time to survival for the restoration of the shortened lower dental arch. $J$ Dent Res 2007;86:646-650.

23. Shahmiri RA, Atieh MA. Mandibular Kennedy Class I implanttooth-borne removable partial denture: A systematic review. J Oral Rehabil 2010;37:225-234.

24. Zhang Q, Kreulen CM, Witter DJ, Creugers NH. Oral health status and prosthodontic conditions of Chinese adults: A systematic review. Int J Prosthodont 2007:20:567-572.

25. Gerritsen AE, Allen PF, Witter DJ, Bronkhorst EM, Creugers $\mathrm{NH}$. Tooth loss and oral health-related quality of life: A systematic review and meta-analysis. Health Qual Life Outcomes 2010;8:126

26. Faggion CM Jr. The shortened dental arch revisited: From evidence to recommendations by the use of the GRADE approach. J Oral Rehabil 2011;38:940-949.

27. Faggion CM Jr, Giannakopoulos NN, Listl S. How strong is the evidence for the need to restore posterior bounded edentulous spaces in adults? Grading the quality of evidence and strength of recommendations. J Dent 2011;39:108-116.

28. Fueki K, Yoshida E, Igarashi Y. A systematic review of prosthetic restoration in patients with shortened dental arches. Jap Dent Sci Rev 2011:47:167-174.

29. Abt E, Carr AB, Worthington HV. Interventions for replacing missing teeth: Partially absent dentition. Cochrane Database Syst Rev 2012;(2):CD003814.

30. Khan S, Musekiwa A, Chikte UME, Omar R. Differences in functional outcomes for adult patients with prosthodonticallytreated and -untreated shortened dental arches: A systematic review. PLoS One 2014:9:e101143.

31. Liang S, Zhang Q, Witter DJ, Wang Y, Creugers NH. Effects of removable dental prostheses on masticatory performance of subjects with shortened dental arches: A systematic review. J Dent 2015:43:1185-1194. 
32. Zeng X, Sheiham A, Tsakos G. Relationship between clinical dental status and eating difficulty in an old Chinese population. J Oral Rehabil 2008;35:37-44.

33. Jepson NJ, Moynihan PJ, Kelly PJ, Watson GW, Thomason JM. Caries incidence following restoration of shortened lower dental arches in a randomized controlled trial. $\mathrm{Br}$ Dent $\mathrm{J}$ 2001;191:140-144.

34. Frank RP, Brudvik JS, Leroux B, Milgrom P, Hawkins N. Relationship between the standards of removable partial denture construction, clinical acceptability, and patient satisfaction. J Prosthet Dent 2000;83:521-527.

35. Cheung A, Weir M, Mayhew A, Kozloff N, Brown K, Grimshaw $J$. Overview of systematic reviews of the effectiveness of reminders in improving healthcare professional behaviour. Syst Rev 2012;1:36.

36. Higgins JPT, Green S. Cochrane Handbook for Systematic Reviews of Interventions, ed 1. West Sussex: Wiley-Blackwell, 2009.

37. Shea BJ, Grimshaw JM, Wells GA, et al. Development of AMSTAR: A measurement tool to assess methodological quality of systematic reviews. BMC Med Res Methodol 2007;7:10.
38. World Medical Organization. Declaration of Helsinki. BMJ 1996;313:1448-1449.

39. Jepson N, Allen F, Moynihan P, Kelly P, Thomason M. Patient satisfaction following restoration of shortened mandibular dental arches in a randomized controlled trial. Int J Prosthodont 2003;16:409-414.

40. Tan H, Peres KG, Peres MA. Do people with shortened dental arches have worse oral health-related quality of life than those with more natural teeth? A population-based study. Community Dent Oral Epidemiol 2015;43:33-46.

41. Sarita PT, Kreulen CM, Witter DJ, van't Hof M, Creugers NH. A study on occlusal stability in shortened dental arches. Int J Prosthod 2003;16:375-380.

42. Kiola IA, Astrøm AN, Strand GV, Masalu JR. Chewing problems and dissatisfaction with chewing ability: A survey of older Tanzanians. Eur J Oral Sci 2007;115:265-274.

43. Creugers $\mathrm{NH}$, Kreulen CM. Systematic review of 10 years of systematic reviews in prosthodontics. Int J Prosthodont 2003;16:123-127.

44. Straus SE, Tetroe J, Graham I. Defining knowledge translation. CMAJ 2009;181:165-168. 